erème, 6,48-6,62. (M M $^{\text {He }} \mathrm{V}$. Lester, Stassano et Rollet, Achard et Stassano.)

Quelques mesures effectuées sur le beurre nous ont donné des chiffres voisins. Nous basons done notre théorie sur des faits expérimentaux et sur la composition chimique du beurre.

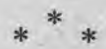

Résumons brièvement ces quelques pages:

Nous avons proposé un nouveau procédé d'analyse bactériologique du beurre. Procédé spécifique pour la recherche des germes du groupe typho-paratyphique.

Ce procédé offre une électivité et une sensibilité encourageantes. Sur 25 beurres analysés à Brest, la proportion de contamination par le bacille d'Eberth était de 4\%.

Enfin, une expérimentation biologique nous a permis de considérer la.vitalité du bacille d'Eberth dans le beurre non pas comme soumise aux variations d'acidité libre du milieu, mais soumise aux variations du $p H$ et fontions du pouvoir-tampon du beurre.

(Laboratoire de Bactériologie, Hôpital maritime de Brest.)

\title{
REVUE
}

\section{LES AGENTS DE NETTOYAGE EMPLOYÉS DANS L'INDUSTRIE LAITIERE}

\author{
par G. GÉNIN
}

Ingénieur E. P. C. I.

Les agents alcalins qui sont utilisés pour le nettoyage des récipients dans l'industrie laitière sont en nombre relativement peu élevé. Arnold H. Johnson, du Laboratoire de Recherches de la "National Dairy Products Corporation ", a étudié, dans un rapport récemment publié, la composition et l'efficacité de ces différents produits.

Les principaux agents alcalins qui sont utilisés en laiterie sont les différentes qualités, que l'on trouve sur le marché, de soude caustique, de métasilicate de sodium, de phosphate trisodique et de carbonate de soude. On trouve dans le commerce, sous des marques de fabrique les plus diverses, ces différents produits, qui sont vendus seuls ou en mélange, additionnés parfois de certains autres constituants. C'est ainsi que lorsque l'agent de nettoyage doit posséder une certaine action abrasive, on lui ajoute une faible quantité de pierre ponce ou un autre produit analogue. On peut également, pour améliorer à un certain point de vue l'efficacité de ces 
agents alcalins, leur ajouter des aluminates, de l'amidon ou des protéines.

Parmi les deux produits qui au cours de ces dernières années ont attiré le plus l'attention des spécialistes, il faut citer l'orthosilicate de sodium et le phosphate disodique. Rappelons que l'orthosilicate est plusalcalin que le métasilicate, tandis que le phosphate disodique est moins alcalin que le phosphate trisodique. Mais en réalité, tous ces produits sont employés en mélange, et d'après JoHnson, qui a étudié en particulier trois d'entre eux, contiennent de 44,5 à $47,5 \%$ de soude caustique et de 63,5 à $67 \%$ d'aleali total. JoHnson a trouvé dans d'autres produits des quantités importantes de phosphate trisodique. Enfin, dans l'un d'entre eux, il a décelé la présence de $66 \%$ de earbonate de soude, de $15 \%$ de phosphate trisodique et une faible proportion de substance colloïdale.

La facilité avec laquelle ces différents agents alcalins dissolvent certains des constituants du lait: protéines, matière grasse, etc., dépend principalement du $p H$ de ces produits lorsqu'ils sont en solution et de la proportion de $\mathrm{Na}^{2} \mathrm{O}$ qu'ils renferment. On sait que les solutions de soude caustique sont celles qui possèdeñt le $p H$ le plus élevé à eoncentration équivalente. Viennent ensuite, dans l'ordre déeroissant, les solutions de métasilicate de sodium, de phosphate trisodique et de carbonate de soude.

Le lavage des bouteilles à lait a pour objet d'assurer une stérilisation pratique de ces récipients, en même temps qu'un lavage purement mécanique. Il y a quelques années, on utilisait plus particulièrement pour cette opération des solutions de 2 à $4 \%$ de soude caustique. Aujourd'hui, la tendance est d'employer de moins en moins de soude caustique et d'opérer à des températures plus élevées. On a constaté en effet que le rinçage des bouteilles lavées à la soude caustique est une opération très difficile. Il semble qu'il adhère à la surface du verre une pellicule alcaline qui n'est pas éliminée par le rinçage. Au contraire, si l'on ajoute à la solution certains sels alealins, le rinçage et l'élimination de la pellicule de soude caustique sont notablement accélérés.

Par suite de ses propriétés détersives excellentes, le métasilicate de sodium a été employé dans de nombreuses machines à laver qui opèrent par trempage. On a toutefois constaté que le manque de propriétés lubrifiantes des solutions de métasilicate de sodium ne permet pas d'obtenir un fonctionnement satisfaisant de ces machines, et, en particulier, les bouteilles ne se déplacent pas facilement sur leur support. L'addition d'une faible quantité de cet agent à la soude caustique permet bien d'obtenir des bouteilles brillantes, mais on a opposé une autre abjection à l'emploi de ce produit, due à ce que les sédiments extraits des bouteilles, au lieu de se déposer 
dans les bacs de lavage, restent en suspension, par suite du caractère colloïdal des sels de calcium qui se forment par réaction du métasilicate de sodium sur les constituants du lait contenant du calcium.

Lorsque, pour le lavage, on emploie des eaux dures, il y a intérêt à ne pas utiliser d'agents détersifs à base de carbonate, car on facilite ainsila formation de dépôts abondants de carbonate de calcium, qui se précipitent dans les appareils sous forme d'un dépôt lourd et embarrassant. $\mathrm{Si}$, au contraire, on emploie du phosphate trisodique ou du métasilicate de sodium avec une eau dure, les précipités qui se forment sont beaucoup moins lourds, de telle sorte qu'ils ne déposent pas et que leur élimination par les eaux de lavage est aisée.

Les sédiments que l'on observe dans les bouteilles à lait ont une composition très variable. Si ces sédiments sont simplement constitués de matière solide, l'application d'une solution alealine à une température comprise entre 50 et $55^{\circ}$ est suffisante pour assurer leur élimination. Si, au contraire, le dépôt provient d'une coagulation des protéines du lait par la ehaleur et peut contenir, en outre, des sels minéraux provenant de l'action des agents de lavage, le traitement par un agent alcalin peut n'être pas suffisant. Dans ce cas, il faut faire appel à l'action d'un acide pour éliminer ces dépôts, qui s'amassent dans des appareils de nettoyage et peuvent gêner leur fonctionnement.

Si la soude caustique additionnée d'un sel alcalin approprié constitue un agent efficace pour le nettoyage des bouteilles, il n'est pas possible de faire appel à ce produit lorsqu'on veut procéder au lavage des appareils métalliques employés en laiterie, par suite de son action corrosive. Par exemple, le fer étamé, ainsi que le cuivre étamé, sont corrodés d'une façon très profonde par la soude caustique libre. Ces mêmes métaux sont également attaqués, mais à un moindre degré, par le carbonate de soude et le phosphate trisodique. Le métasilicate de sodium est l'agent le moins corrosif de tous les métaux étamés. Toutefois, lorsque les conditions sont favorables, il peut attaquer l'étain. L'aluminium est profondément corrodé par la soude eaustique, le phosphate trisodique et le carbonate de soude. Le laiton, le bronze, ont leur surface ternie par l'action des alcalis; le nickel et le métal Monel sont très résistants à l'action de ces produits et, enfin, les aciers à $18 \%$ de chrome et à $8 \%$ de nickel sont parfaitement résistants.

Lorsqu'on ehoisira done un agent destiné au nettoyage des appareils et des bouteilles employés en laiterie, on n'oubliera pas de faire intervenir dans ce choix les conditions d'emploi de cet agent, ainsi que la nature des matériaux auxquels il doit s'appliquer. 